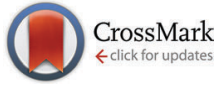

Cite this: Phys. Chem. Chem. Phys., 2015, 17, 18547

Received 22nd April 2015, Accepted 11th June 2015

DOI: $10.1039 / c 5 c p 02349 a$

www.rsc.org/pccp

\title{
Development and applications of the LFDFT: the non-empirical account of ligand field and the simulation of the $f-d$ transitions by density functional theory + t
}

\author{
Harry Ramanantoanina, ${ }^{\star a}$ Mohammed Sahnoun, ${ }^{\mathrm{b}}$ Andrea Barbiero, ${ }^{\mathrm{a}}$ \\ Marilena Ferbintean $u^{c}$ and Fanica Cimpoesu ${ }^{* d}$
}

\begin{abstract}
Ligand field density functional theory (LFDFT) is a methodology consisting of non-standard handling of DFT calculations and post-computation analysis, emulating the ligand field parameters in a non-empirical way. Recently, the procedure was extended for two-open-shell systems, with relevance for inter-shell transitions in lanthanides, of utmost importance in understanding the optical and magnetic properties of rare-earth materials. Here, we expand the model to the calculation of intensities of $f \rightarrow d$ transitions, enabling the simulation of spectral profiles. We focus on $\mathrm{Eu}^{2+}$-based systems: this lanthanide ion undergoes many dipole-allowed transitions from the initial $4 f^{7}\left({ }^{8} S_{7 / 2}\right)$ state to the final $4 f^{6} 5 d^{1}$ ones, considering the free ion and doped materials. The relativistic calculations showed a good agreement with experimental data for a gaseous $\mathrm{Eu}^{2+}$ ion, producing reliable Slater-Condon and spin-orbit coupling parameters. The $\mathrm{Eu}^{2+}$ ion-doped fluorite-type lattices, $\mathrm{CaF}_{2}: \mathrm{Eu}^{2+}$ and $\mathrm{SrCl}_{2}: \mathrm{Eu}^{2+}$, in sites with octahedral symmetry, are studied in detail. The related Slater-Condon and spin-orbit coupling parameters from the doped materials are compared to those for the free ion, revealing small changes for the $4 \mathrm{f}$ shell side and relatively important shifts for those associated with the $5 \mathrm{~d}$ shell. The ligand field scheme, in Wybourne parameterization, shows a good agreement with the phenomenological interpretation of the experiment. The non-empirical computed parameters are used to calculate the energy and intensity of the $4 f^{7}-4 f^{6} 5 d^{1}$ transitions, rendering a realistic convoluted spectrum.
\end{abstract}

\section{Introduction}

The concept of the ligand field, very fruitful in the effective account of bonding and properties in coordination chemistry, is equivalent to the crystal field theory in condensed matter science. Both terminologies refer to the same phenomenological model, operated with adjustable parameters.

\footnotetext{
${ }^{a}$ Department of Chemistry of the University of Fribourg (Switzerland), Chemin du Musée 9, 1700 Fribourg, Switzerland. E-mail: harry.ra@hotmail.com; Fax: +4126300 9738; Tel: +41263008700

${ }^{b}$ Laboratoire de Physique de la Matière et Modélisation Mathématique, LPQ3M, Université de Mascara, Algeria

${ }^{c}$ Faculty of Chemistry, Inorganic Chemistry Department, University of Bucharest, Dumbrava Rosie 23, Bucharest 0206462, Romania

${ }^{d}$ Institute of Physical Chemistry, Splaiul Independentei 202, Bucharest 060021, Romania.E-mail: cfanica@yahoo.com

$\dagger$ Dedicated to Professor Claude Daul and Professor Werner Urland in the celebration of their seventieth and seventy-first anniversaries.

\$ Electronic supplementary information (ESI) available: The whole range of the spectral energy obtained for $\mathrm{CaF}_{2}: \mathrm{Eu}^{2+}$ and $\mathrm{SrCl}_{2}: \mathrm{Eu}^{2+}$ together with a direct comparison between the theoretical spectra and the excitation spectra in ref. 41 and 42. See DOI: $10.1039 / \mathrm{c} 5 \mathrm{cp} 02349 \mathrm{a}$
}

Born more than eighty years ago, from the work of $\mathrm{H}$. Bethe ${ }^{1}$ and J. H. van Vleck $^{2}$ it still keeps the position of the most transparent way to describe the optical and magnetic properties of metal ion-based systems (lattices or molecular complexes). As long as quantum chemical methods can compute reliable energy level schemes, the subsequent ligand field analysis of the raw results is the way to illuminate in depth the underlying mechanism. ${ }^{3-5}$ Stricto sensu, the ligand field refers to effective one-electron parameters accounting for the effect of the environment on a metal ion, but the complete frame includes the interelectron effects, describing the electronic correlation in the active space of $\mathrm{d}^{n}$ or $\mathrm{f}^{n}$ configurations, and also the spin-orbit coupling, namely the relativistic effects. Besides the standard theory, one must note the paradigm shift due to C. E. Schäffer and C. K. Jørgensen, who revisited the ligand field theory to ensure more chemical insight within their Angular Overlap Model (AOM), initially devoted to the d-type transition metal systems. ${ }^{6} \mathrm{~W}$. Urland pioneered this model for the f-type ligand field, in lanthanide compounds, with convincing applications in spectroscopy and magnetism. ${ }^{7}$

About two decades ago, given the important growth of computational techniques, the demand for a predictive theory compatible 
with the classical formalism of the ligand field theory emerged. In particular, this is not a trivial task in the frame of density functional theory (DFT), limited to non-degenerate ground states, while ligand field concerns the full multiplets originating from $\mathrm{d}^{n}$ or $\mathrm{f}^{n}$ configurations. In the consistent solving of this problem, C. Daul erat primus. He and co-workers (noting the contribution of $\mathrm{M}$. Atanasov) designed a pioneering approach by non-routine handling of DFT numeric experiments, to extract ligand field parameters, in a post-computational algorithm named LFDFT. ${ }^{8-10}$ The procedure treats the near degeneracy correlation explicitly within the model space of the Kohn-Sham orbitals possessing dominant $\mathrm{d}$ and $\mathrm{f}$ characters.

In LFDFT, the basic start is a DFT calculation performed in average of configuration (AOC) conditions. Namely, for a given $\mathrm{d}^{n}$ (or $\mathrm{f}^{n}$ ) configuration of the metal ion in the complex, the occupation of five (or, respectively, seven) Kohn-Sham orbitals carrying main d (or f) character is fixed to the general fractional $n / 5$ (or $n / 7$, respectively) numbers. This corresponds to the barycentre conceived in formal ligand field theories. Subsequently, with the converged AOC orbitals, a series of numeric experiments are done, producing the configurations related to the distribution of $n$ electrons in the five (or seven) orbitals identified as the ligand field sequence (this time with corresponding integer populations). These determinant configurations are not real states, but useful computational experiments, able to render ligand field parameters. The situation is somewhat similar to broken symmetry treatments, ${ }^{1-14}$ where the spin-polarized configurations cannot be claimed as physical states, but artificial constructions relevant for the emulation of the exchange coupling parameters. ${ }^{15}$ Then, the LFDFT run of different configurations based on AOC orbitals yields ligand field parameters, altogether with inter-electron Coulomb and exchange effective integrals. Thus, the Slater determinants are used as the basis in the computational model. In the advanced background of the theory, a canonical number of configurations needed to reproduce the desired parameters can be defined as a function of the symmetry of the problem (Slater determinant wavefunctions of spin-orbitals weighted by symmetry coefficients). ${ }^{10}$ In practice, the full set of configurations can be generated, performing the least square fit relating the computed energy expectation values against the ligand field model formulas. The obtained parameters are further used in setting configuration interaction (CI) matrices, in the spirit of the ligand field formalism, sustained in a non-empirical manner. Therefore C. Daul et al. have realized the parameterfree ligand field theory, which became a valuable tool for any consideration of multiplet states in DFT.
We recognize herein the impact of the LFDFT in solving various electronic structure problems. This computational gadget has revolutionized many fields of chemical science, being applied in theoretical investigations ${ }^{16-20}$ as well as in experimental works. ${ }^{21,22}$

A priori, LFDFT has determined the multiplet energy levels within an accuracy of a few hundred wavenumbers. ${ }^{23}$ The model has given satisfactory results for the molecular properties arising from a single-open-shell system, such as zero-field splitting (ZFS), ${ }^{24,25}$ magnetic exchange coupling, ${ }^{26-29}$ Zeeman interaction, ${ }^{30}$ hyper-fine splitting, ${ }^{30}$ shielding constants, ${ }^{31,32}$ $\mathrm{d}-\mathrm{d}$ and $\mathrm{f}-\mathrm{f}$ transitions. ${ }^{10,17,33,34}$

Recently, the LFDFT algorithm has been updated to handle the electronic structure of two-open-shell systems, as it is important in the understanding of the optical manifestation of lanthanide phosphors. ${ }^{35,36}$ Lanthanide compounds are agents in lightemitting diode (LED) technology, used in domestic lighting. ${ }^{37}$ In the case of a two-open-shell inter-configuration of $\mathrm{f}$ and $\mathrm{d}$ electrons, the size of the ligand field CI matrices is collected in Table 1, calculated with the following combinatorial formulas:

$$
\begin{gathered}
N\left(4 \mathrm{f}^{n}\right)=\left(\begin{array}{l}
14 \\
n
\end{array}\right), \\
N\left(4 \mathrm{f}^{n-1} 5 \mathrm{~d}^{1}\right)=\left(\begin{array}{l}
14 \\
n-1
\end{array}\right) \cdot\left(\begin{array}{l}
10 \\
1
\end{array}\right),
\end{gathered}
$$

as a function of the number of active electrons (n). We can confine to a single $\mathrm{f}-\mathrm{d}$ orbital promotion, since the energy of two and further electron processes is too high. It is seen from Table 1 that the size of the CI matrices increases drastically, for some cases ( $n=7$ or 8 ) a parallelized algorithm having been required to achieve calculations.

In this paper, we present new development and applications of the LFDFT algorithm, previously validated for the two-openshell $4 \mathrm{f}^{1} 5 \mathrm{~d}^{1}$ electronic structure of $\operatorname{Pr}^{3+} \cdot{ }^{35,38-40}$ Special attention will be paid to $\mathrm{Eu}^{2+}$ systems, i.e. for $n=7$ (Table 1), taking as examples divalent europium doped in the fluorite-type lattices $\mathrm{CaF}_{2}$ and $\mathrm{SrCl}_{2}$, comparing the first principles results with the available experimental data. ${ }^{41,42}$

\section{Methodology}

The two-open-shell ligand field-based CI Hamiltonian in eqn (3) combines quantum effects due to the inter-electron repulsion and exchange $\left(H_{\mathrm{EE}}\right)$, the spin-orbit coupling $\left(H_{\mathrm{SO}}\right)$ and the ligand field effective one-electron $\left(H_{\mathrm{LF}}\right):^{35}$

$$
H=H_{0}+H_{\mathrm{EE}}+H_{\mathrm{SO}}+H_{\mathrm{LF}}
$$

\begin{tabular}{|c|c|c|c|c|c|c|c|c|c|c|c|c|c|c|}
\hline$n$ & 1 & 2 & 3 & 4 & 5 & 6 & 7 & 8 & 9 & 10 & 11 & 12 & 13 & 14 \\
\hline $\left.4 f^{n}\right)$ & 14 & 91 & 364 & 1001 & 2002 & 3003 & 3432 & 3003 & 2002 & 1001 & 364 & 91 & 14 & 1 \\
\hline$N\left(4 \mathrm{f}^{n-1} 5 \mathrm{~d}^{1}\right)$ & 10 & 140 & 910 & 3640 & 10010 & 20020 & 30030 & 34320 & 30030 & 20020 & 10010 & 3640 & 910 & 140 \\
\hline$\sum^{a}$ & 24 & 231 & 1274 & 4641 & 12012 & 23023 & 33462 & 37323 & 32032 & 21021 & 10374 & 3731 & 924 & 141 \\
\hline
\end{tabular}

Table 1 Total number of generated Slater determinants corresponding to the $4 f^{n}$ and $4 f^{n-1} 5 d^{1}$ electron configurations of lanthanide ions with $n$ valence electrons

${ }^{a} \sum$ represents the cumulative sum of $N\left(4 \mathrm{f}^{n}\right)$ and $N\left(4 \mathrm{f}^{n-1} 5 \mathrm{~d}^{1}\right)$. 
where $H_{0}$ is a diagonal matrix, which gathers contributions of zeroth-order interactions, such as the kinetic energy background and the nuclear-electron attraction of the AOC configuration:

$$
H_{0}=-\frac{\hbar^{2}}{2 m} \sum_{i} \nabla_{i}^{2}-\sum_{i} \frac{Z e^{2}}{r_{i}} \text {. }
$$

This term acts only on the diagonal of the full ligand field CI matrix:

$$
H_{0}=\left(\begin{array}{ll}
I_{N\left(4 \mathrm{f}^{n}\right)} \cdot 0 & (0) \\
(0) & I_{N\left(4 \mathrm{f}^{n-1} 5 \mathrm{~d}^{1}\right)} \cdot \Delta(\mathrm{fd})
\end{array}\right)
$$

where $I_{N}$ is an identity matrix of dimension $N$ (see Table 1) and $\Delta(\mathrm{fd})$ is the gap parameter, which determines the energy difference between the barycentre of the multiplet levels of the excited $4 \mathrm{f}^{n-1} 5 \mathrm{~d}^{1}$ and of those of the ground $4 \mathrm{f}^{n}$ electron configuration. Therefore, in further consideration, the $H_{\mathrm{EE}}, H_{\mathrm{SO}}$ and $H_{\mathrm{LF}}$ matrices are simply traceless blocks, their possible diagonal elements already engulfed in $\Delta(\mathrm{fd})$.

The matrix elements of $H_{\mathrm{EE}}$ are constructed from the twoelectron integrals:

$$
\left\langle\psi_{\mathrm{a}} \psi_{\mathrm{b}}\left|H_{\mathrm{EE}}\right| \psi_{\mathrm{c}} \psi_{\mathrm{d}}\right\rangle=\int \psi_{\mathrm{a}}{ }^{*}\left(r_{1}\right) \psi_{\mathrm{b}}{ }^{*}\left(r_{2}\right) \frac{1}{r_{12}} \psi_{\mathrm{c}}\left(r_{1}\right) \psi_{\mathrm{d}}\left(r_{2}\right) \mathrm{d} r_{1} \mathrm{~d} r_{2},
$$

where $\psi$ denotes the atomic orbital wavefunctions:

$$
\psi(r)=R_{\mathrm{nl}}(r) Y_{\operatorname{lm}}(\theta, \phi),
$$

$R_{\mathrm{nl}}$ is the radial wavefunction of the atomic shell and $Y_{\mathrm{lm}}$ is the spherical harmonic component. It is a basic assumption of the ligand field framework that the two-electron part can be treated like in the free atom. ${ }^{35}$

Within mathematical operations, eqn (6) is reducible into the product of two integrals of angular and radial components. Once the angular part is explicitly resolved, the whole variety of the eqn (6) integrals can be represented by a few radial SlaterCondon parameters, $F_{k}$ (eqn (8) and (9)) and $G_{k}$ (eqn (10)), with intra- or inter-shell nature. In the two-open-shell problem of $4 \mathrm{f}$ and $5 \mathrm{~d}$ electrons, one obtains:

$$
\begin{gathered}
F_{k}(\mathrm{ff})=\int_{0}^{\infty} \int_{0}^{\infty} \frac{r_{<}^{k}}{r_{>}^{k+1}} R_{4 \mathrm{f}^{2}}\left(r_{1}\right) R_{4 \mathrm{f}}{ }^{2}\left(r_{2}\right) r_{1}{ }^{2} r_{2}{ }^{2} \mathrm{~d} r_{1} \mathrm{~d} r_{2} \\
F_{k}(\mathrm{fd})=\int_{0}^{\infty} \int_{0}^{\infty} \frac{r_{<}^{k}}{r_{>}^{k+1}} R_{4 \mathrm{f}}{ }^{2}\left(r_{1}\right) R_{5 \mathrm{~d}}{ }^{2}\left(r_{2}\right) r_{1}{ }^{2} r_{2}{ }^{2} \mathrm{~d} r_{1} \mathrm{~d} r_{2} \\
G_{k}(\mathrm{fd})=\int_{0}^{\infty} \int_{0}^{\infty} \frac{r_{<}^{k}}{r_{>}^{k+1}} R_{4 \mathrm{f}}\left(r_{1}\right) R_{5 \mathrm{~d}}\left(r_{2}\right) R_{5 \mathrm{~d}}\left(r_{1}\right) R_{4 \mathrm{f}}\left(r_{2}\right) r_{1}{ }^{2} r_{2}{ }^{2} \mathrm{~d} r_{1} \mathrm{~d} r_{2} .
\end{gathered}
$$

The matrix elements of $H_{\text {So }}$ express the spin-orbit structure of the electronic multiplets. The formulation of $H_{\text {So }}$ has been the subject of numerous investigations ${ }^{3,43-45}$ where its matrix elements have been reasonably well approximated in atomiclike integrals:

$$
\left\langle n l s m_{1} m_{s}\left|H_{\mathrm{SO}}\right| n l s^{\prime} m_{1}^{\prime} m_{s}{ }^{\prime}\right\rangle=\zeta_{\mathrm{nl}}\left\langle l s m_{1} m_{s}|\hat{l} . \hat{s}| l s^{\prime} m_{1}{ }^{\prime} m_{s}{ }^{\prime}\right\rangle,
$$

where $\zeta_{n l}$ is the effective one-electron spin-orbit coupling constants for one electron in a $n l$ atomic shell. It can be analytically evaluated using the radial wavefunction $R_{n l}$ of the atomic shell:

$$
\zeta_{n l}=\frac{Z e^{2} \hbar^{2}}{8 \pi \varepsilon_{0} m_{0}^{2} c^{2}}\left\langle R_{n l}\left|\frac{1}{r^{3}}\right| R_{n l}\right\rangle .
$$

The matrix elements of $H_{\mathrm{LF}}$ play the role of the chemical environment of the lanthanide ion. The general formulation of the ligand field potential follows Wybourne: ${ }^{46}$

$$
\begin{aligned}
& \left\langle l_{\mathrm{a}} m_{\mathrm{la}}\left|H_{\mathrm{LF}}\right| l_{\mathrm{b}} m_{\mathrm{lb}}\right\rangle \\
& =\sum_{k=0}^{l_{\mathrm{a}}+l_{\mathrm{b}}} \sum_{q=-k}^{k} B_{q}^{k}\left(l_{\mathrm{a}}, l_{\mathrm{b}}\right)\left\langle Y_{l_{\mathrm{a}} m_{\mathrm{la}}}(\theta, \phi)\left|C_{q}^{(k)}(\theta, \phi)\right| Y_{l_{\mathrm{b}} m_{\mathrm{lb}}}(\theta, \phi)\right\rangle,
\end{aligned}
$$

where $C_{q}^{(k)}$ represent the solid spherical harmonic tensor operators (eqn (11)) and $B_{q}^{k}$ are the Wybourne-normalized crystal field parameters;

$$
C_{q}^{(k)}=\sqrt{\frac{4 \pi}{2 k+1}} Y_{k q} .
$$

The collection of non-vanishing Wybourne parameters depends on the coordination symmetry of the lanthanide centre, their total number in a two-open-shell $\mathrm{f}-\mathrm{d}$ ligand field problem being 64 in the case of the $C_{1}$ point group. ${ }^{47}$ Here, they cannot be reduced to having a simple electrostatic origin, since the DFT calculation takes into consideration different effects including orbital overlap and covalence. ${ }^{35}$

Besides the Hamiltonian setting, other specific construction regards the matrix element of the dipole moment operator, important to the computation of the intensity of transitions:

$$
\left\langle\psi_{\mu}\left|\vec{d}_{\alpha}\right| \psi_{\nu}\right\rangle=\frac{1}{\sqrt{3}}\left\langle R_{n_{\mu} l_{\mu}}|r| R_{n_{\nu} l_{\nu}}\right\rangle\left\langle Y_{l_{\mu} m_{\mu}}\left|C_{\alpha}^{(1)}\right| Y_{l_{\nu} m_{\nu}}\right\rangle
$$

where, in the right hand side of eqn (15), the term carrying the radial component is simple overlap integrals, while the angular term is proportional to Clebsch-Gordan coefficients. ${ }^{39}$ Actually, only the $\mathrm{f}-\mathrm{d}$ elements are non-vanishing, their mutual mixing by ligand field rendering the intensity, in an approximate, but apparently satisfactory manner.

In summary, several series of parameters have to be determined non-empirically in order to perform LFDFT calculations of twoopen-shell $\mathrm{f}$ and $\mathrm{d}$ electrons:

(1) $\Delta(\mathrm{fd})$, which represents the energy shift of the multiplets of the $4 \mathrm{f}^{n-1} 5 \mathrm{~d}^{1}$ configuration with respect to those of the $4 \mathrm{f}^{n}$ configuration.

(2) $F_{k}(\mathrm{ff}), F_{k}(\mathrm{fd})$ and $G_{k}(\mathrm{fd})$, which represent the static electron correlation within the $4 \mathrm{f}^{n}$ and $4 \mathrm{f}^{n-1} 5 \mathrm{~d}^{1}$ configurations.

(3) $\zeta_{n l}$, which represents the relativistic spin-orbit interaction in the $4 \mathrm{f}$ and $5 \mathrm{~d}$ shells.

(4) $B_{q}^{k}(\mathrm{f}, \mathrm{f}), B_{q}^{k}(\mathrm{~d}, \mathrm{~d})$ and $B_{q}^{k}(\mathrm{f}, \mathrm{d})$, which describe the interaction due to the presence of the ligands onto the electrons of the metal centre.

The DFT calculations have been carried out by means of the Amsterdam density functional (ADF) program package (ADF2013.01). ${ }^{48-50}$ We must point out that the ADF is one of 
the few DFT codes that has the set of keywords facilitating the AOC calculations and Slater determinant emulation, needed by the LFDFT procedure. ${ }^{35,36}$ The hybrid B3LYP functional ${ }^{51}$ was used to compute the electronic structure and the related optical properties, in line with previous works. ${ }^{35,36,39}$ The molecular orbitals were expanded using triple-zeta plus two polarization Slater-type orbital (STO) functions (TZ2P+) for the Eu atom and triple-zeta plus one polarization STO function (TZP) for the Ca, $\mathrm{Sr}, \mathrm{F}$ and $\mathrm{Cl}$ atoms.

The geometrical structures due to the doping of the $\mathrm{Eu}^{2+}$ ion into $\mathrm{CaF}_{2}$ and $\mathrm{SrCl}_{2}$ lattices were approached via periodical calculations by means of the VASP program package. ${ }^{52}$ The local density approximation (LDA) defined in the Vosko-WilkNusair $(\mathrm{VWN})^{53}$ and the generalized gradient approximation (GGA) outlined in the Perdew-Burke-Ernzerhof $(\mathrm{PBE})^{54}$ were used for the exchange-correlation functional. The interaction between valence and core electrons was emulated with the projected augmented wave method. ${ }^{55,56}$ External as well as semi-core states were included in the valence. A plane-waves basis set with a cut-off energy of $400 \mathrm{eV}$ was used. Super-cells representing a 2 by 2 by 2 expansion of the unit cells of $\mathrm{CaF}_{2}$ and $\mathrm{SrCl}_{2}$ were simulated, which were found to be large enough to lead to negligible interactions between the periodic images of the $\mathrm{Eu}^{2+}$ impurity. $4 k$-points were included in each direction of the lattice. The atomic positions were allowed to relax until all forces were smaller than $0.005 \mathrm{eV}^{-1}$.

\section{Results and discussion}

\section{The determination of $\Delta(\mathrm{fd})$}

We must discuss at the very beginning the calculation of the $\Delta(\mathrm{fd})$ gap, which is important in the problem of two-open-shell systems because it sets the origin of the energy of the two electron configurations, conventionally 0 for the $4 \mathrm{f}^{n}$ and $\Delta(\mathrm{fd})$ for the $4 \mathrm{f}^{n-1} 5 \mathrm{~d}^{1}$. In a first description, we work with the free ion, considering the gaseous $\mathrm{Eu}^{2+}$ case. Estimating by DFT the energy difference between the $4 \mathrm{f}^{6} 5 \mathrm{~d}^{1}$ and $4 \mathrm{f}^{7}$ configurations, we must work under the AOC references. This is because the $\Delta(\mathrm{fd})$ is not the difference between the specific energy levels, but rather a gap of the averaged energy values common for all multiplets of $4 \mathrm{f}^{n}$ and $4 \mathrm{f}^{n-1} 5 \mathrm{~d}^{1}$ kinds.

$\mathrm{T}$. Ziegler et al. clarified early that the occupation-averaged configurations, called transition states, carry in DFT the meaning of statistically-averaged spectral terms. ${ }^{57}$ We prepare the wavefunctions $\psi_{4 \mathrm{f}}$ and $\psi_{5 \mathrm{~d}}$ by AOC where six and one electrons are evenly distributed in the $4 \mathrm{f}$ and $5 \mathrm{~d}$ orbitals of $\mathrm{Eu}^{2+}$, respectively (Fig. 1). This will generate the reference totally symmetric density, which will be used to compute the DFT energy associated with the series of Slater determinants. Thus all the Slater determinant energies are successively computed permuting seven electrons in the $4 \mathrm{f}$ wavefunction (Fig. 1) for the $4 \mathrm{f}^{7}$ manifold, and permuting six electrons in the $4 \mathrm{f}$ wavefunction plus one electron in the $5 \mathrm{~d}$ for the $4 \mathrm{f}^{6} 5 \mathrm{~d}^{1}$ manifold. The results obtained at the B3LYP level of theory are graphically represented in Fig. 2 showing the $\Delta(\mathrm{fd})$ gap. Note that $\Delta(\mathrm{fd})$ can

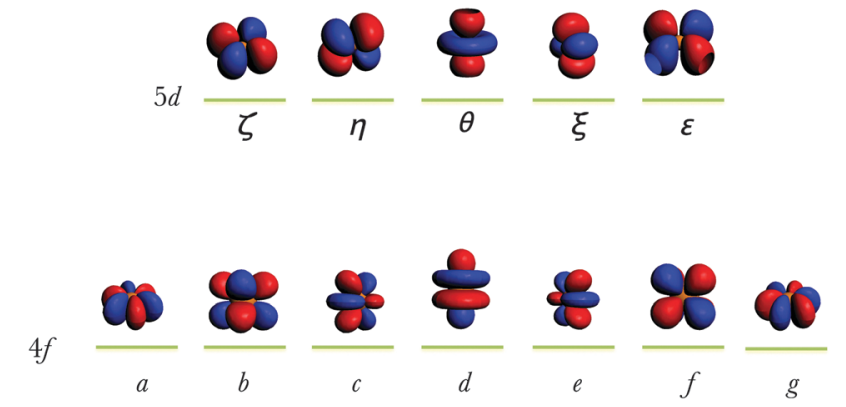

Fig. 1 Representation of the $\psi_{4 \mathrm{f}}$ and $\psi_{5 \mathrm{~d}}$ orbitals of $\mathrm{Eu}^{2+}$, obtained from an $\mathrm{AOC}$ calculation of $\mathrm{Eu}^{2+}$ within the $4 \mathrm{f}^{6} 5 \mathrm{~d}^{1}$ electron configuration. The components of the $4 f$ orbitals are listed from left to right according to: $f_{x\left(x^{2}-3 y^{2}\right)}, f_{x y z}, f_{z^{2} x}, f_{z^{3}}, f_{z^{2} y}, f_{z\left(x^{2}-y^{2}\right)}$ and $f_{y\left(3 x^{2}-y^{2}\right)}$, i.e. $a, b, c, d, e, f$ and $g$. The components of the $5 \mathrm{~d}$ orbitals are listed from left to right according to: $d_{x y}, d_{x z}, d_{z^{2}}, d_{y z}$ and $d_{x^{2}-y^{2}}$, i.e. $\zeta, \eta, \theta, \xi$ and $\varepsilon$.

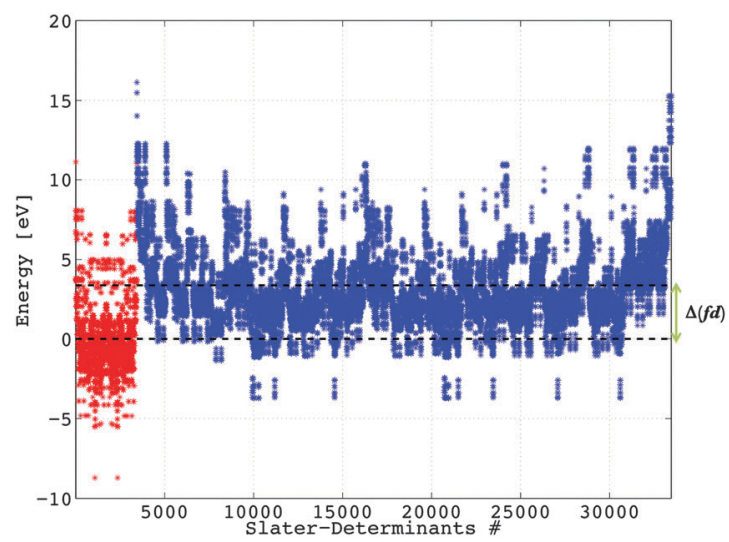

Fig. 2 Representation of the calculated DFT energy values associated with the 3432 Slater determinants (in red) arising from the $4 f^{7}$ and the 30030 Slater determinants (in blue) arising from the $4 f^{6} 5 d^{1}$ configurations of $\mathrm{Eu}^{2+}$. The two dashed lines represent the barycentre of the $4 \mathrm{f}^{7}$ manifold (set to the zero of energy), and that of the $4 f^{6} 5 d^{1}$ manifold.

occasionally have a negative value, indicating that the ground electron configuration of the lanthanide ion is $4 \mathrm{f}^{n-1} 5 \mathrm{~d}^{1}$ instead of $4 \mathrm{f}^{n}$. Such a situation may appear in the case of lanthanide $\mathrm{Gd}^{2+}(n=8$, see Table 1$)$ and $\mathrm{La}^{2+}(n=1$, see Table 1$)$ ions.

The lowest energies corresponding to the $4 \mathrm{f}^{7}$ manifold (Fig. 2) are associated with the Slater determinants:

$$
\left|\mathrm{a}^{+} \mathrm{b}^{+} \mathrm{c}^{+} \mathrm{d}^{+} \mathrm{e}^{+} \mathrm{f}^{+} \mathrm{g}^{+}\right| \text {and }\left|\mathrm{a}^{-} \mathrm{b}^{-} \mathrm{c}^{-} \mathrm{d}^{-} \mathrm{e}^{-} \mathrm{f}^{-} \mathrm{g}^{-}\right|
$$

where the signs + and - represent the spin of one electron, up or down, respectively. The highest energies corresponding to the $4 f^{6} 5 d^{1}$ manifold (Fig. 2) are associated with the Slater determinants:

$$
\left|\mathrm{c}^{ \pm} \mathrm{d}^{ \pm} \mathrm{e}^{ \pm} \theta^{+}\right| \text {and }\left|\mathrm{c}^{ \pm} \mathrm{d}^{ \pm} \mathrm{e}^{ \pm} \theta^{-}\right|
$$

where the sign \pm represents a restricted occupation of two electrons in one orbital. The calculated value of the $\Delta(\mathrm{fd})$ parameter is $3.10 \mathrm{eV}$ at the B3LYP level of theory.

The DFT Slater determinant energies (Fig. 2) can also provide information about the two-electron $F_{k}(\mathrm{ff}), F_{k}(\mathrm{fd})$ and $G_{k}(\mathrm{fd})$ parameters using Slater's rule ${ }^{3}$ and least mean square fitting. ${ }^{10}$ 
However, this procedure might undergo uncertainty caused by the important number of linear equations versus variables. In the case of two-open-shell $4 \mathrm{f}^{7}$ and $4 \mathrm{f}^{6} 5 \mathrm{~d}^{1}$ of $\mathrm{Eu}^{2+}$, for instance, it returns to solve 33462 linear equations with nine variables, leading to some misrepresentations of the parameters. ${ }^{58}$ Therefore, we calculate the $F_{k}(\mathrm{ff}), F_{k}(\mathrm{fd})$ and $G_{k}(\mathrm{fd})$ parameters from the radial wavefunctions $R_{\mathrm{nl}}$ of the $4 \mathrm{f}$ and $5 \mathrm{~d}$ Kohn-Sham orbitals of the lanthanide ions following eqn (8)-(10), which is the subject of the next section.

\section{The calculation of the $F_{k}, G_{k}$, and $\zeta_{n 1}$ parameters}

The importance of relativity in the physics of lanthanide elements is not negligible. ${ }^{59-63}$ There are different approaches dedicated to the implementation of relativistic corrections in DFT. Besides the spinorbit interaction $H_{\mathrm{SO}}$ (eqn (11)), which has itself a relativistic origin; the physics behind the Dirac equation in quantum chemistry is reasonably well characterized by the scalar-relativistic equations. ${ }^{64}$ We can perform scalar-relativistic calculations at the zerothorder regular approximation (ZORA) to the Dirac equation ${ }^{65-69}$ or the first-order relativistic Pauli Hamiltonian ${ }^{70-74}$ in DFT. In Fig. 3, the solutions of the radial wavefunctions of the $4 \mathrm{f}$ and $5 \mathrm{~d}$ KohnSham orbitals obtained for a gaseous $\mathrm{Eu}^{2+}$ ion are graphically represented, where the influence of the relativistic correction is evaluated. A noticeable expansion of the $R_{\mathrm{nl}}$ is observed when relativistic corrections are implemented in the computational details (Fig. 3), in line with the definition of relativity acting on f and d orbitals. ${ }^{64,75}$ This expansion is severely pronounced for the Pauli-relativistic calculation (Fig. 3), because of the explicit insertion of the Darwin and mass-velocity terms in the master equation. ${ }^{70-74}$ The calculated $F_{k}(\mathrm{ff}), F_{k}(\mathrm{fd}), G_{k}(\mathrm{fd})$ and $\zeta_{\mathrm{nl}}$ parameters using $R_{4 \mathrm{f}}$ and $R_{5 \mathrm{~d}}$ (Fig. 3) are collected in Table 2. In total, there are: ${ }^{35}$

three $F_{k}(\mathrm{ff})$ parameters: $F_{2}(\mathrm{ff}), F_{4}(\mathrm{ff})$ and $F_{6}(\mathrm{ff})$; plus

two $F_{k}(\mathrm{fd})$ parameters: $F_{2}(\mathrm{fd})$ and $F_{4}(\mathrm{fd})$; plus

three $G_{k}(\mathrm{fd})$ parameters: $G_{1}(\mathrm{fd}), G_{3}(\mathrm{fd})$ and $G_{5}(\mathrm{fd})$; plus

two spin-orbit coupling constants: $\zeta_{4 \mathrm{f}}$ and $\zeta_{5 \mathrm{~d}}{ }^{35}$

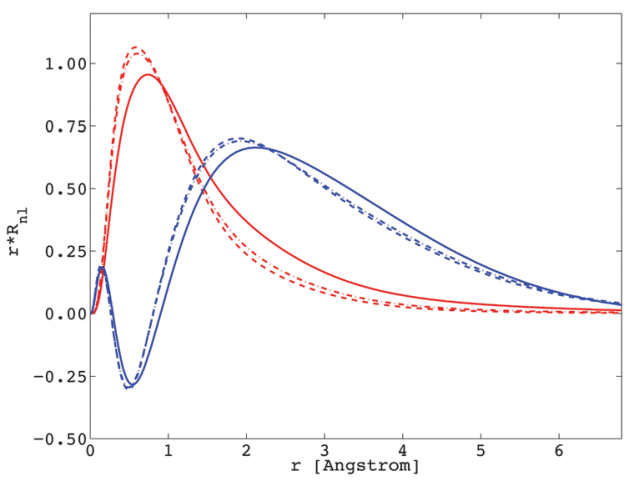

Fig. 3 Representation of the radial wavefunctions $R_{\mathrm{nl}}$ corresponding to the $4 \mathrm{f}$ (in red) and $5 \mathrm{~d}$ (in blue) Kohn-Sham orbitals of a gaseous $\mathrm{Eu}^{2+}$ ion, obtained at the Pauli-relativistic (solid curve), the ZORA-relativistic (dotted-and-dashed curve) and the non-relativistic (dashed curve) levels of theory.
Table 2 Calculated Slater-Condon parameters and spin-orbit coupling constants (in $\mathrm{cm}^{-1}$ ) obtained at the non-relativistic (a), the ZORA-relativistic (b) and the Pauli-relativistic (c) levels of theory, corresponding to the twoopen-shell $4 f^{7}$ and $4 f^{6} 5 d^{1}$ electron configurations of a gaseous $E u^{2+}$ ion

Slater-Condon parameters and spin-orbit coupling constants (a)

\begin{tabular}{lr}
\hline$F_{2}(\mathrm{ff})$ & 500.19 \\
$F_{4}(\mathrm{ff})$ & 64.66 \\
$F_{6}(\mathrm{ff})$ & 6.87 \\
$F_{2}(\mathrm{fd})$ & 245.32 \\
$F_{4}(\mathrm{fd})$ & 17.86 \\
$G_{1}(\mathrm{fd})$ & 338.38 \\
$G_{3}(\mathrm{fd})$ & 29.97 \\
$G_{5}(\mathrm{fd})$ & 4.70 \\
$\zeta_{4 \mathrm{f}}$ & 2133.90 \\
$\zeta_{5 \mathrm{~d}}$ & 1279.31
\end{tabular}

(b)

$\begin{array}{rr}475.60 & 388.47 \\ 61.32 & 49.92 \\ 6.51 & 5.30 \\ 245.36 & 244.72 \\ 18.12 & 18.82 \\ 369.81 & 431.92 \\ 31.66 & 35.34 \\ 4.91 & 5.40 \\ 1980.90 & 1246.50 \\ 1245.93 & 987.25\end{array}$

The parameters in Table 2 were determined from the wavefunctions $\psi_{4 \mathrm{f}}$ and $\psi_{5 \mathrm{~d}}$, prepared in the same manner as was done for Fig. 1.

Note that the parameters $F_{2}(\mathrm{ff}), F_{4}(\mathrm{ff})$ and $F_{6}(\mathrm{ff})$ are acting principally on the single-open-shell $4 \mathrm{f}^{n}$ configuration, but they are also present in the diagonal block of the $4 \mathrm{f}^{n-1} 5 \mathrm{~d}^{1}$ interaction matrix. Experimentally known spectral terms of the $4 \mathrm{f}^{7}$ configuration of $\mathrm{Eu}^{2+}$ concern only the ground state ${ }^{8} \mathrm{~S}$ and the two excited states ${ }^{6} \mathrm{P}$ and ${ }^{6} \mathrm{I},{ }^{76}$ although there are 119 levels arising from the multi-electron configuration. ${ }^{77}$ The calculated energy values of these ${ }^{8} \mathrm{~S},{ }^{6} \mathrm{P}$ and ${ }^{6} \mathrm{I}$ spectral terms are given in Table 3 , obtained using the parameters in Table 2. They are also compared with the available experimental data taken from the framework of the NIST atomic spectra database. ${ }^{76}$

We determined the deviations between the calculated and the experimental spectral terms (Table 3) using eqn (16):

$$
\varepsilon[\%]=100 \times \frac{\left|E_{\mathrm{calc}}-E_{\mathrm{exp}}\right|}{E_{\exp }} .
$$

For the three computational methods under consideration, we obtained a maximum deviation of $30.64 \%, 24.09 \%$ and $2.41 \%$ for the non-relativistic, ZORA-relativistic and Pauli-relativistic

Table 3 Calculated multiplet energy levels (calc.) of a gaseous $\mathrm{Eu}^{2+}$ ion (in $\mathrm{cm}^{-1}$ ) at non-relativistic (a), ZORA-relativistic (b) and Pauli-relativistic (c) levels of theory, compared with the experimentally-known spectral terms (exp.) corresponding to the $4 f^{7}$ electron configuration

\begin{tabular}{lrrrr}
\hline & \multicolumn{2}{l}{ Calc. } & & \\
\cline { 2 - 4 } & \multicolumn{1}{l}{ (a) } & (b) & (c) & \multicolumn{1}{c}{ Exp. $^{a}$} \\
\hline${ }^{8} \mathrm{~S}_{7 / 2}$ & 0.00 & 0.00 & 0.00 & 0.00 \\
${ }^{6} \mathrm{P}_{7 / 2}$ & 36379.05 & 34596.14 & 28854.94 & 28200.06 \\
${ }^{6} \mathrm{P}_{5 / 2}$ & 37400.88 & 35526.15 & 29317.04 & 28628.54 \\
${ }^{6} \mathrm{P}_{3 / 2}$ & 38339.68 & 36381.98 & 29758.39 & $-{ }^{a}$ \\
${ }^{6} \mathrm{I}_{7 / 2}$ & 40277.33 & 38282.44 & 31591.89 & 31745.99 \\
${ }^{6} \mathrm{I}_{9 / 2}$ & 40978.41 & 38917.65 & 31888.23 & 31954.21 \\
${ }^{6} \mathrm{I}_{17 / 2}$ & 41370.71 & 39274.11 & 32060.93 & 32073.30 \\
${ }^{6} \mathrm{I}_{11 / 2}$ & 41542.51 & 39430.07 & 32135.09 & 32179.55 \\
${ }^{6} \mathrm{I}_{15 / 2}$ & 41901.08 & 39756.07 & 32293.32 & 32307.78 \\
${ }^{6} \mathrm{I}_{13 / 2}$ & 41881.85 & 39739.21 & 32287.83 & 32314.14
\end{tabular}

${ }^{a}$ Taken from ref. 76 where the energy value of ${ }^{6} \mathrm{P}_{3 / 2}$ is not known. 
calculations, respectively. On the other hand, we also obtained a minimum deviation of $26.87 \%, 20.59 \%$ and $0.04 \%$. There is an appropriate agreement between the Pauli-relativistic results and the experimental data. Experimentally-known spectral terms of the $4 \mathrm{f}^{6} 5 \mathrm{~d}^{1}$ configuration of $\mathrm{Eu}^{2+}$ in ref. 76 assemble some states of octet and sextet spin multiplicity, which in tensor operator techniques represent the direct product: $4 \mathrm{f}^{6}\left({ }^{7} \mathrm{~F}\right) \otimes{ }^{2} \mathrm{D}$, giving rise to the following terms: ${ }^{8} \mathrm{P},{ }^{6} \mathrm{P},{ }^{8} \mathrm{D},{ }^{6} \mathrm{D},{ }^{8} \mathrm{~F},{ }^{6} \mathrm{~F},{ }^{8} \mathrm{G},{ }^{6} \mathrm{G},{ }^{8} \mathrm{H}$ and ${ }^{6} \mathrm{H}$. Note that the whole manifold of the $4 \mathrm{f}^{6} 5 \mathrm{~d}^{1}$ configuration allows the consideration of 906 spectral terms, including not only the high octet spin multiplicity but also the lower sextet, quartet and doublet, the energies of which are obtained from the DFT calculation using the $F_{k}(\mathrm{ff}), F_{k}(\mathrm{fd}), G_{k}(\mathrm{fd}), \zeta_{4 \mathrm{f}}$ and $\zeta_{5 \mathrm{~d}}$ parameters (Table 2) and the $\Delta(\mathrm{fd})$ parameter discussed in the previous section.

The calculated deviations $\varepsilon$ (eqn (16)) from the experimentallyknown spectral terms ${ }^{76}$ are represented in Fig. 4 for the three theoretical methods under consideration. Here also the Paulirelativistic calculation leads to the best reproduction of the experimental data, its mean deviation being $6.17 \%$ (Fig. 4), which is far smaller than those obtained at the non-relativistic and ZORA-relativistic levels of theory.

In this section, the impact of the relativistic correction on the spectroscopy of lanthanide ions is clearly justified; an appropriate description of the radial $R_{4 \mathrm{f}}$ and $R_{5 \mathrm{~d}}$ wavefunctions is a prerequisite, enabling a good reproduction of the experimental data.

\section{Structural analysis of the doped systems}

The doping of lanthanide ions into solid state materials is nowadays a topic of significant interest due to the optical effects. ${ }^{37}$ There are several instrumental methods to probe the local structure around the impurity ions in solid state compounds, such as nuclear magnetic resonance (NMR), ${ }^{78}$ extended X-ray absorption fine structure (EXAFS), ${ }^{79,80}$ as well as electron paramagnetic resonance (EPR). ${ }^{81}$ However, these methods do not give direct results of the local geometry, offering only data that can be corroborated to it. A clear answer is found in the theoretical side, mimicking the doping of solid state materials by means of

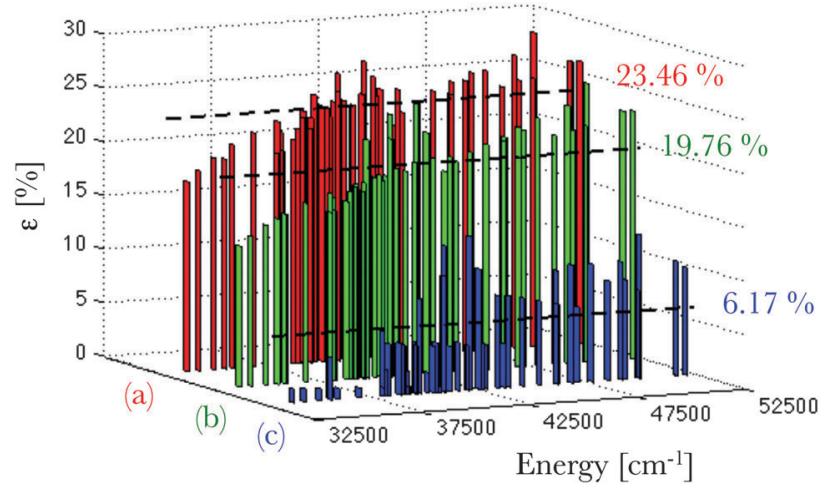

Fig. 4 Representation of the error distribution $\varepsilon$ (in \%) with respect to the experimental data ${ }^{76}$ of the calculated multiplet energy levels corresponding to the $4 f^{6} 5 d^{1}$ configuration of a gaseous $\mathrm{Eu}^{2+}$ ion, at the nonrelativistic (in red, (a)), ZORA-relativistic (in green, (b)) and Pauli-relativistic (in blue, (c)) levels of theory. The calculated mean deviations from the experimental data are also given. band structure methods. In this section, we investigate the local structure around the $\mathrm{Eu}^{2+}$ impurity incorporated in the $\mathrm{CaF}_{2}$ and $\mathrm{SrCl}_{2}$ matrices. Calcium fluoride $\left(\mathrm{CaF}_{2}\right)$ and strontium chloride $\left(\mathrm{SrCl}_{2}\right)$ belong to the cubic $F m \overline{3} m$ space group (No. 225). ${ }^{41,42}$ The divalent $\mathrm{Eu}^{2+}$ ion enters in the matrices in the site formally occupied by $\mathrm{Ca}^{2+}$ and $\mathrm{Sr}^{2+}$. It is then coordinated by eight fluoride or chloride ligands, respectively, in the systems $\mathrm{CaF}_{2}: \mathrm{Eu}^{2+}$ and $\mathrm{SrCl}_{2}: \mathrm{Eu}^{2+}$, within the $O_{\mathrm{h}}$ point group.

For the pristine $\mathrm{CaF}_{2}$ and $\mathrm{SrCl}_{2}$ systems (Fig. 5), the calculated lattice parameters are given in Table 4 in terms of the DFT functional used in the band structure algorithm. It is found that both GGA and LDA calculations yield different lattice equilibrium constants (Table 4), i.e. different local relaxations. In terms of a direct comparison, we consider the GGA calculation most appropriate to simulate the experimental data, although the cells are slightly larger than the experimental ones.

For the $\mathrm{CaF}_{2}: \mathrm{Eu}^{2+}$ and $\mathrm{SrCl}_{2}: \mathrm{Eu}^{2+}$ systems, we constructed super-cells which double the number of the unit cells of $\mathrm{CaF}_{2}$ and $\mathrm{SrCl}_{2}$ in the $a, b$ and $c$ directions. The $\mathrm{Eu}^{2+}$ ion was placed in the position $(0,0,0)$. In these cases, the super-cells are big enough inasmuch as the interactions between two $\mathrm{Eu}^{2+}$ ions are minimized. We relaxed the positions of the atoms, fixing the lattice parameters to the theoretical values obtained for the pure systems. This mimics the resistance of the whole lattice against defect-induced distortions, under the conditions of a lower doping concentration than the $2 \times 2 \times 2$ super-cells actually worked upon have. The optimized $\mathrm{Eu}-\mathrm{F}$ and $\mathrm{Eu}-\mathrm{Cl}$ bond lengths are $2.4732 \AA$ and $3.0774 \AA$, respectively, which represent an elongation with respect to the $\mathrm{Ca}-\mathrm{F}$ and $\mathrm{Sr}-\mathrm{Cl}$ bond lengths obtained for the pure systems: $2.3893 \AA$ and $3.0515 \AA$ A.
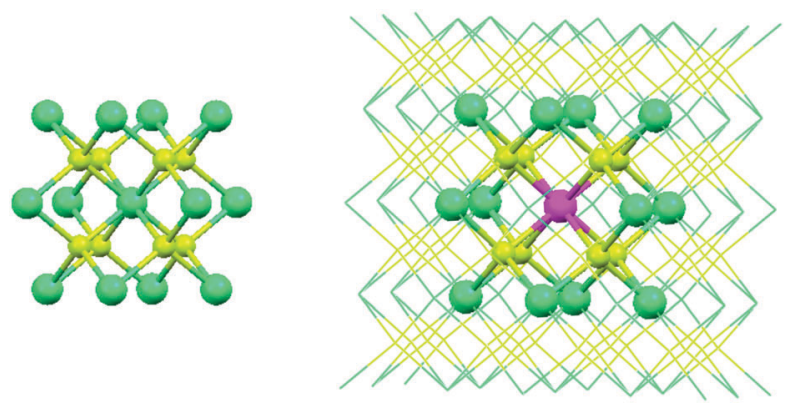

Fig. 5 Representation of the crystal structure of $\mathrm{CaF}_{2}$ showing the unit cell (left hand side). The local structure of an $\mathrm{Eu}^{2+}$ centre embedded in a $2 \times 2 \times 2$ unit cell of $\mathrm{CaF}_{2}$ (right hand side). Colour code: $\mathrm{Ca}^{2+}$ in green, $\mathrm{F}^{-}$ in yellow and $\mathrm{Eu}^{2+}$ in violet. For clarity, some $\mathrm{Ca}^{2+}$ and $\mathrm{F}^{-}$ions are represented with a wireframe shape.

Table 4 Calculated lattice parameters $a, b, c$ (in $\AA$ ) and $\alpha, \beta, \gamma$ (in $\AA$ ) obtained for $\mathrm{CaF}_{2}$ and $\mathrm{SrCl}_{2}$ crystallizing in the cubic $\mathrm{Fm} \overline{3} \mathrm{~m}$ space group (No. 225), compared with experimental X-ray diffraction data

\begin{tabular}{cccccccc}
\hline & $\mathrm{CaF}_{2}$ & & & & $\mathrm{SrCl}_{2}$ & & \\
\cline { 2 - 4 } & $\mathrm{LDA}$ & $\mathrm{GGA}$ & Exp. $^{a}$ & & LDA & GGA & Exp. $^{b}$ \\
\hline$a, b, c$ & 5.3342 & 5.5179 & 5.4355 & 6.8088 & 7.0472 & 6.965 \\
$\alpha, \beta, \gamma$ & 90.0 & 90.0 & 90.0 & & 90.0 & 90.0 & 90.0
\end{tabular}

${ }^{a}$ Taken from ref. 82. ${ }^{b}$ Taken from ref. 83. 
The description of the local structure of doped materials is important in the further evaluation of the ligand field Hamiltonian (eqn (13)), the presence of the impurity in the host materials producing distortions due to differences in the ionic radii or electronic structure. We favoured here the band structure algorithms for geometrical purposes, although we can certainly conceive of a cluster geometry optimization approach, which is already popular in computational chemistry, especially while dealing with excited states geometry. ${ }^{36,39}$

\section{The calculation of $B_{q}^{k}$ ligand field parameters}

In the $\mathrm{CaF}_{2}: \mathrm{Eu}^{2+}$ and $\mathrm{SrCl}_{2}: \mathrm{Eu}^{2+}$ systems, the site symmetry of the $\mathrm{Eu}^{2+}$ impurity is $O_{\mathrm{h}}$ and the non-zero Wybourne parameters are as follows:

$$
B_{0}^{4}(\mathrm{f}, \mathrm{f}), B_{4}^{4}(\mathrm{f}, \mathrm{f}), B_{-4}^{4}(\mathrm{f}, \mathrm{f}), B_{0}^{6}(\mathrm{f}, \mathrm{f}), B_{4}^{6}(\mathrm{f}, \mathrm{f}) \text { and } B_{-4}^{6}(\mathrm{f}, \mathrm{f}),
$$

for the sub-matrix corresponding to the $\left\langle\mathrm{f}\left|H_{\mathrm{LF}}\right| \mathrm{f}\right\rangle$ (eqn (13));

$$
B_{0}^{4}(\mathrm{~d}, \mathrm{~d}), B_{4}^{4}(\mathrm{~d}, \mathrm{~d}) \text { and } B_{-4}^{4}(\mathrm{~d}, \mathrm{~d}),
$$

for the sub-matrix corresponding to the $\left\langle\mathrm{d}\left|H_{\mathrm{LF}}\right| \mathrm{d}\right\rangle$ (eqn (13)).

The inversion center in the $O_{\mathrm{h}}$ point group allows vanishing of the elements of the sub-matrix $\left\langle\mathrm{f}\left|H_{\mathrm{LF}}\right| \mathrm{d}\right\rangle .^{47}$

The ligand field energy schemes of the $4 \mathrm{f}$ and the $5 \mathrm{~d}$ orbitals of $\mathrm{Eu}^{2+}$ in the $\mathrm{CaF}_{2}: \mathrm{Eu}^{2+}$ and $\mathrm{SrCl}_{2}: \mathrm{Eu}^{2+}$ systems were calculated taking the cubic clusters $\left(\mathrm{EuF}_{8}\right)^{6-}$ and $\left(\mathrm{EuCl}_{8}\right)^{6-}$, respectively, which have the optimized geometries obtained in the previous section. Point charges were placed at the coordinates of the next-neighbouring $\mathrm{Ca}^{2+}$ and $\mathrm{Sr}^{2+}$ ions, which are also shown as ball-and-sticks in the super-cell in Fig. 5. These were used in order to mimic the long-range interaction of the crystal hosts.

The ligand field energies and wavefunctions were obtained from Kohn-Sham orbitals of restricted DFT calculations within the AOC reference, by evenly placing six electrons in the $4 \mathrm{f}$ orbitals and one electron in the $5 \mathrm{~d}$. We previously presented the analysis of the ligand field interaction with respect to the change of the DFT functional for the two-open-shell $4 \mathrm{f}$ and $5 \mathrm{~d}$ problem in $\operatorname{Pr}^{3+} .{ }^{35}$ It was found that, in the $5 \mathrm{~d}$ ligand field, the DFT functional does not play an important role, whereas in the $4 \mathrm{f}$, the hybrid B3LYP functional is required in order to obtain realistic ligand field parameters. ${ }^{35}$ Therefore we have used B3LYP for the computation of the electronic structure of $\mathrm{Eu}^{2+}$.

The $4 \mathrm{f}$ orbitals form the basis of $\mathrm{t}_{1 \mathrm{u}}, \mathrm{t}_{2 \mathrm{u}}$ and $\mathrm{a}_{2 \mathrm{u}}$ irreducible representations (irreps) of the $O_{\mathrm{h}}$ point group. The $5 \mathrm{~d}$ orbitals are in the basis of the $e_{g}$ and the $t_{2 g}$ irreps. The values of the ligand field $B_{q}^{k}$ parameters were determined by linear equation fitting using eqn (13), knowing the following ratios for the octahedral symmetry constraint:

$$
B_{4}^{4}(1,1)=B_{-4}^{4}(1,1)=\sqrt{\frac{5}{14}} B_{0}^{4}(1,1),
$$

with $l$ standing for $\mathrm{d}$ and $\mathrm{f}$, and

$$
B_{4}^{6}(\mathrm{f}, \mathrm{f})=B_{-4}^{6}(\mathrm{f}, \mathrm{f})=-\sqrt{\frac{7}{2}} B_{0}^{6}(\mathrm{f}, \mathrm{f})
$$

The calculated values of the $B_{q}^{k}$ parameters for the $\mathrm{CaF}_{2}: \mathrm{Eu}^{2+}$ and $\mathrm{SrCl}_{2}: \mathrm{Eu}^{2+}$ systems are presented in Table 5, together with
Table 5 Calculated ligand field parameters (calc.) in $\mathrm{cm}^{-1}$ obtained for the systems $\mathrm{CaF}_{2}: \mathrm{Eu}^{2+}$ and $\mathrm{SrCl}_{2}: \mathrm{Eu}^{2+}$, compared with available experimental data (exp.)

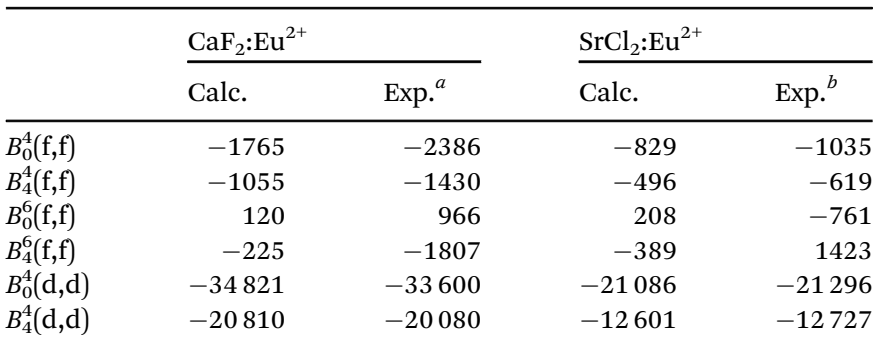

${ }^{a}$ Taken from ref. $41{ }^{b}$ Taken from ref. 42.

the experimentally-deduced ones. For $B_{q}^{k}(\mathrm{~d}, \mathrm{~d})$, the theoretical values are in good agreement with the experimental data. ${ }^{41,42}$ However, for $B_{q}^{k}(\mathrm{f}, \mathrm{f})$, although the $B_{0}^{4}(\mathrm{f}, \mathrm{f})$ and ipso facto the $B_{4}^{4}(\mathrm{f}, \mathrm{f})$ (eqn (17)) are also in the magnitude of the experimental data, the $B_{0}^{6}(\mathrm{f}, \mathrm{f})$ and related parameters (eqn (18)) are slightly underestimated for both the $\mathrm{CaF}_{2}: \mathrm{Eu}^{2+}$ and $\mathrm{SrCl}_{2}: \mathrm{Eu}^{2+}$ systems. This departure between the calculated and the experimental values is reflected primarily in the ordering of the 4 f orbitals splitting. The ordering obtained from computation was as follows for both $\mathrm{CaF}_{2}: \mathrm{Eu}^{2+}$ and $\mathrm{SrCl}_{2}: \mathrm{Eu}^{2+}$ systems:

$$
\mathrm{t}_{1 \mathrm{u}}<\mathrm{t}_{2 \mathrm{u}}<\mathrm{a}_{2 \mathrm{u}}
$$

while it resulted in the swapped sequence,

$$
\mathrm{t}_{1 \mathrm{u}}<\mathrm{a}_{2 \mathrm{u}}<\mathrm{t}_{2 \mathrm{u}}
$$

for the experimentally-deduced parameters obtained for the $\mathrm{SrCl}_{2}: \mathrm{Eu}^{2+}$ system. ${ }^{42}$

The change in the orbital ordering may be attributed to the impact of the neighbouring cations, where the symmetry-adapted linear combination of their virtual orbitals may stabilize the $a_{2 u}$ irrep. This is not achieved here in the small cluster models of $\left(\mathrm{EuCl}_{8}\right)^{6-}$. Nevertheless, a direct comparison between $B_{q}^{k}(\mathrm{f}, \mathrm{f})$ and $B_{q}^{k}(\mathrm{~d}, \mathrm{~d})$ indicates that the effect of the $4 \mathrm{f}$ parameters will be completely superseded by the $5 \mathrm{~d}$ ones.

\section{AOM analysis of the ligand field interaction}

For the sake of more intuitive insight, the $B_{q}^{k}$ parameters can be converted to the AOM scheme ${ }^{47}$ reformulating the ligand field matrix in eqn (13) as follows:

$$
\begin{aligned}
\left\langle 3, m\left|H_{\mathrm{LF}}\right| 3, m^{\prime}\right\rangle & =\sum_{k=1}^{\text {ligands }} \sum_{\lambda=\sigma, \pi} D_{m, \lambda}^{4 \mathrm{f}}(k) \cdot D_{m^{\prime}, \lambda}^{4 \mathrm{f}}(k) \cdot e_{\lambda, k}(4 \mathrm{f}), \\
\left\langle 2, m\left|H_{\mathrm{LF}}\right| 2, m^{\prime}\right\rangle & =\sum_{k=1}^{\text {ligands }} \sum_{\lambda=\sigma, \pi} D_{m, \lambda}^{5 \mathrm{~d}}(k) \cdot D_{m^{\prime}, \lambda}^{5 \mathrm{~d}}(k) \cdot e_{\lambda, k}(5 \mathrm{~d}),
\end{aligned}
$$

where $D^{4 \mathrm{f}}$ and $D^{5 \mathrm{~d}}$ are the matrix elements defined in terms of Euler angles (Wigner's Darstellungsmatrizen) $)^{6,7,47}$ and $k$ is the running index for the ligand system. The $e_{\lambda} \equiv e_{\sigma}, e_{\pi}$ parameters have the meaning of perturbation exerted by $\sigma$ and $\pi$ sub-components of 
the density cloud of the ligands (or by corresponding overlap effects, in another heuristic formulation).

A general problem in establishing the parametric conversion is the fact that the AOM matrix is not traceless, the sum of the diagonal elements for a homoleptic $\left[\mathrm{ML}_{n}\right]$ complex with linearly ligating ligands (isotropic $\pi$ effects) being $n\left(e_{\sigma}+2 e_{\pi}\right)$, instead of zero, like in the standard ligand field model. In the case of the $4 \mathrm{f}$ shell, in octahedral symmetry, the situation does not impinge upon the parametric conversion since we have two independent parameters, $B_{0}^{4}(\mathrm{f}, \mathrm{f})$ and $B_{0}^{6}(\mathrm{f}, \mathrm{f})$ in the Wybourne scheme (Table 5), versus two AOM parameters $e_{\sigma}(4 \mathrm{f})$ and $e_{\pi}(4 \mathrm{f})$, uniquely related to the two relative gaps in the ligand field splitting in $O_{\mathrm{h}}$ symmetry.

The mutual conversion is done by the following formulas:

$$
\begin{aligned}
& e_{\sigma}(4 \mathrm{f})=-\frac{9}{44} B_{0}^{4}(\mathrm{f}, \mathrm{f})+\frac{63}{1144} B_{0}^{6}(\mathrm{f}, \mathrm{f}) \\
& e_{\pi}(4 \mathrm{f})=-\frac{3}{22} B_{0}^{4}(\mathrm{f}, \mathrm{f})-\frac{189}{1144} B_{0}^{6}(\mathrm{f}, \mathrm{f}) .
\end{aligned}
$$

The comparison of computed versus experimental fitted Wybourne parameters (Table 5) can be regarded as semi-quantitative in general, with certain apparent mismatches, as is the opposite sign found for the $B_{0}^{6}(\mathrm{f}, \mathrm{f})$ value in the case of the $\mathrm{SrCl}_{2}: \mathrm{Eu}^{2+}$ system. The same sign mismatch in the case of the computed versus fitted $B_{4}^{6}(\mathrm{f}, \mathrm{f})$ is not an independent feature, given the mentioned $B_{4}^{6}(\mathrm{f}, \mathrm{f}) / B_{0}^{6}(\mathrm{f}, \mathrm{f})$ proportionality (eqn (18)). The conversion to AOM parameterization allows a certain assessment of the situation. Thus, the calculated AOM parameters for the $\mathrm{SrCl}_{2}: \mathrm{Eu}^{2+}$ system are as follows (in $\left.\mathrm{cm}^{-1}\right): e_{\sigma}(4 \mathrm{f})=181.02$ and $e_{\pi}(4 \mathrm{f})=78.68$, while the conversion of reported fitted $B_{0}^{4}(\mathrm{f}, \mathrm{f})$ and $B_{0}^{6}(\mathrm{f}, \mathrm{f})$ values $^{42}$ yielded the following values: $e_{\sigma}(4 \mathrm{f})=169.79$ and $e_{\pi}(4 \mathrm{f})=266.86$. One observes that the experimental values lead to the somewhat counterintuitive situation of $e_{\pi}(4 \mathrm{f})>e_{\sigma}(4 \mathrm{f})$ values, with the result then that the numeric experiment may be, in a relative sense, a more reliable source, not for absolute values but with respect to the inter-parametric ratios. The fact is that the ligand field parameters on the $4 \mathrm{f}$ shell show small values, in general, being prone to fit uncertainties given the large number of active parameters. The reference work ${ }^{42}$ considered a fit with several empirical terms such as Trees and Marvin corrections, while keeping imposed fixed ratios among the more fundamental Slater-Condon parameters, and therefore the full comparability of computed versus fitted parameters is partly hindered, considering that we worked here only by first-principle leverages: ligand field, Slater-Condon and spin-orbit coupling parameters, without other degrees of freedom.

For the $5 \mathrm{~d}$ shell, the single gap between $\mathrm{e}_{\mathrm{g}}$ and $\mathrm{t}_{2 \mathrm{~g}}$ does not need the two AOM parameters, so that must impose certain conventions, like the $e_{\sigma}(5 \mathrm{~d}) / e_{\pi}(5 \mathrm{~d})=3$ ratio. ${ }^{35}$ However, we have not advanced in this direction, given the good match of the computed and fitted 5d-type $B_{q}^{k}$ parameters, which do not demand the call of AOM as a further moderator in the comparative discussion.

The ligand field interaction, besides lifting the degeneracy of the $4 \mathrm{f}$ and $5 \mathrm{~d}$ orbitals, also has a side effect expanding the radial wavefunctions towards the ligands positions. This is commonly known as the nephelauxetic effect, a concept coined by C. K. Jørgensen ${ }^{84}$ which is the subject of the next section.

\section{The nephelauxetic effect}

The nephelauxetic effect describes the fact that the parameter values of the inter-electron repulsion are usually smaller in complexes than in the corresponding free ions. ${ }^{84}$ The word nephelauxetic was created by basic translation of "cloud expansion" from Greek. We can quantitatively analyze the changes in the metal wavefunctions with respect to the presence of ligands, underlying the action of the nephelauxetic effect. The uf shell is shielded from the interaction with the chemical environment inasmuch as independently to the ligand type, the reduction of the free ion inter-electron repulsion $F_{k}(\mathrm{ff})$ parameters are negligible. ${ }^{47,85}$ On the other hand, the virtual $5 \mathrm{~d}$ and $6 \mathrm{~s}$ shells are able to interact with the neighbourhood, ensuring therefore the bonding of lanthanide ions. ${ }^{86-88}$ We present in Fig. 6 the radial wavefunction $R_{5 \mathrm{~d}}$ of $\mathrm{Eu}^{2+}$ in the presence of eight fluoride and eight chloride ligands in a cubic arrangement. For comparison purposes, we also present the radial wavefunction obtained for the gaseous $\mathrm{Eu}^{2+}$ free ion (Fig. 3). One observes the pronounced expansion of $R_{5 \mathrm{~d}}$ in the series of fluoride and chloride ligands, highlighting the overlap of ligands by the orbitals from the lanthanide ion. Due to this effect, as explained in previous instances, in the excited states of a $4 \mathrm{f}^{n-1} 5 \mathrm{~d}^{1}$ lanthanide configuration, the calculated bond lengths are always shorter than those obtained in the ground $4 \mathrm{f}^{n}$ configuration. ${ }^{36,39}$

Recalling eqn (9) and (10), we calculated the $F_{k}(\mathrm{fd})$ and $G_{k}(\mathrm{fd})$ parameters in the complex, based on the radial shapes shown in Fig. 6. Compared with Fig. 3, one notes that $R_{4 \mathrm{f}}$ remains almost the same, while $R_{5 \mathrm{~d}}$ were shifted by the nephelauxetic effect (see also ref. 47).

The results are given in Table 6, together with the calculated spin-orbit coupling constant $\zeta_{5 \mathrm{~d}}$, using eqn (12) and the $\Delta(\mathrm{fd})$ gap. All the parameters (Table 6) are reduced when compared to the Pauli-relativistic quantities in Table 2 . The nephelauxetic ratio $\beta$ is defined as the fraction made from the inter-electron

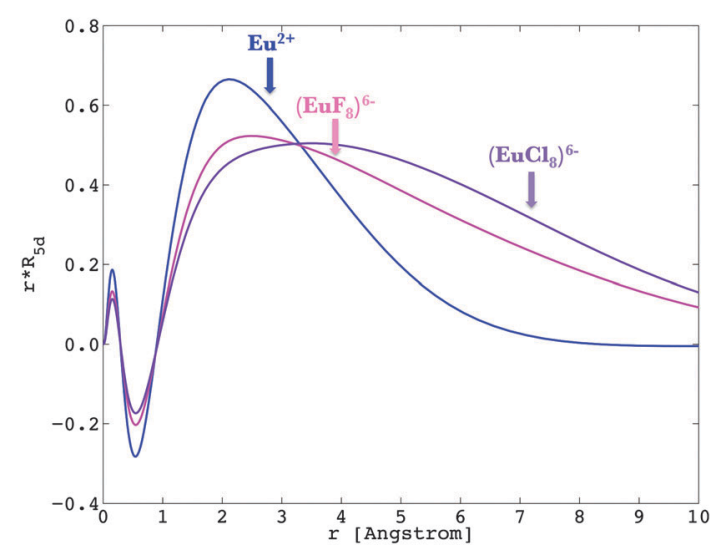

Fig. 6 Representation of $R_{5 \mathrm{~d}}$ of $\mathrm{Eu}^{2+}$ in the free ion (in blue), in $\left(\mathrm{EuF}_{8}\right)^{6-}$ (in pink) and $\left(\mathrm{EuCl}_{8}\right)^{6-}$ (in violet), obtained at the Pauli-relativistic level of theory. 
Table 6 Calculated values of the Slater-Condon $F_{k}(\mathrm{fd})$ and $G_{k}(\mathrm{fd})$, the spin-orbit coupling $\zeta_{5 d}$ and the $\Delta(\mathrm{fd}) \mathrm{gap}$ (in $\mathrm{cm}^{-1}$ ) obtained for the systems $\mathrm{CaF}_{2}: \mathrm{Eu}^{2+}$ and $\mathrm{SrCl}_{2}: \mathrm{Eu}^{2+}$, compared with the experimentallydeduced values

\begin{tabular}{|c|c|c|c|c|c|c|}
\hline & \multicolumn{3}{|l|}{$\mathrm{CaF}_{2}: \mathrm{Eu}^{2+}$} & \multicolumn{3}{|l|}{$\underline{\mathrm{SrCl}_{2}: \mathrm{Eu}^{2+}}$} \\
\hline & Calc. & $\beta$ & Exp. $^{a}$ & Calc. & $\beta$ & Exp. $^{a}$ \\
\hline$F_{2}(\mathrm{fd})$ & 138.42 & 0.57 & 133.33 & 100.56 & 0.41 & 117.43 \\
\hline$F_{4}(\mathrm{fd})$ & 9.88 & 0.53 & 10.25 & 6.79 & 0.36 & 8.54 \\
\hline$G_{1}(\mathrm{fd})$ & 232.08 & 0.54 & 192.29 & 160.56 & 0.37 & 162.06 \\
\hline$G_{3}(\mathrm{fd})$ & 18.22 & 0.52 & 17.30 & 12.31 & 0.35 & 14.41 \\
\hline$G_{5}(\mathrm{fd})$ & 2.74 & 0.51 & 2.72 & 1.84 & 0.34 & 2.26 \\
\hline$\zeta_{5 \mathrm{~d}}$ & 505.76 & 0.51 & 760 & 371.14 & 0.38 & 844 \\
\hline$\Delta(\mathrm{fd})$ & 18800 & - & 23500 & 12400 & - & - \\
\hline
\end{tabular}

parameters obtained in the complex and in the free ion, for instance:

$$
\beta\left(F_{2}(\mathrm{fd})\right)=\frac{F_{2}(\mathrm{fd})_{\text {Complex }}}{F_{2}(\mathrm{fd})_{\text {Free ion }}} .
$$

The calculated $\beta$ values for $F_{k}(\mathrm{fd}), G_{k}(\mathrm{fd})$ and $\zeta_{5 \mathrm{~d}}$ were also given in Table 5. We calculate mean $\beta$ values of 0.53 and 0.37 for $\mathrm{CaF}_{2}: \mathrm{Eu}^{2+}$ and $\mathrm{SrCl}_{2}: \mathrm{Eu}^{2+}$, respectively.

We can obtain the $\Delta(\mathrm{fd})$ gap for the $\mathrm{CaF}_{2}: \mathrm{Eu}^{2+}$ system from ref. 41 which we compare with our calculated value (Table 6). Unfortunately, the experimental value for the same parameter was not specified for the $\mathrm{SrCl}_{2}: \mathrm{Eu}^{2+}$ system. ${ }^{42}$ The difference between the calculated $\Delta(\mathrm{fd})$ gap and that obtained in ref. 41 is directly related to the $F_{k}(\mathrm{ff})$ parameters (Table 2), which is also present in the diagonal elements of the CI matrix of the $4 \mathrm{f}^{6} 5 \mathrm{~d}^{1}$ configuration of $\mathrm{Eu}^{2+}$. Since the values of our calculated $F_{k}(\mathrm{ff})$ parameters are larger than that given in ref. 41 our $\Delta(\mathrm{fd})$ is accordingly smaller.

\section{The $4 f^{7}-4 f^{6} 5 d^{1}$ transitions}

The multiplet energy levels corresponding to the $4 \mathrm{f}^{7}$ and the $4 \mathrm{f}^{6} 5 \mathrm{~d}^{1}$ configurations of $\mathrm{Eu}^{2+}$ doped into $\mathrm{CaF}_{2}$ and $\mathrm{SrCl}_{2}$ are given in Fig. 7 in the spectral range of $0-55000 \mathrm{~cm}^{-1}$ (those for the entire spectral range are given in the ESI, $\ddagger$ Fig. S1 and S2). They were computed in the LFDFT algorithm using the following non-empirical parameters: $F_{k}(\mathrm{ff})$ and $\zeta_{4 \mathrm{f}}$ (Table 2$) ; F_{k}(\mathrm{fd})$,

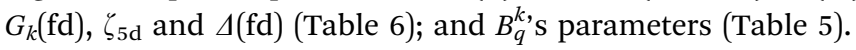

The transitions from the initial $4 \mathrm{f}^{7}\left({ }^{8} \mathrm{~S}_{7 / 2}\right)$ state to the final $4 \mathrm{f}^{6} 5 \mathrm{~d}^{1}$ are electric dipole-allowed, with the calculation of the electric dipole transition moments obtained from eqn (15). The oscillator strength for the zero phonon lines between the ground state ${ }^{8} S_{7 / 2}$ of $4 f^{7}$ and the final states of $4 f^{6} 5 d^{1}$ are calculated and represented in Fig. 7. The most intense transitions are given with respect to the irreps of the octahedral double group. In the circumstances of a non-degenerate ${ }^{8} \mathrm{~S}_{7 / 2}$ state of the $4 \mathrm{f}^{7}$ subsystem, the energies of the $4 \mathrm{f}^{7}-4 \mathrm{f}^{6} 5 \mathrm{~d}^{1}$ transitions are practically the same as the position of $4 \mathrm{f}^{6} 5 \mathrm{~d}^{1}$ spectral terms. The intensities were computed by corresponding handling of the dipole moment represented in the ligand field CI basis, depending all on a single reduced matrix element, ultimately irrelevant as an
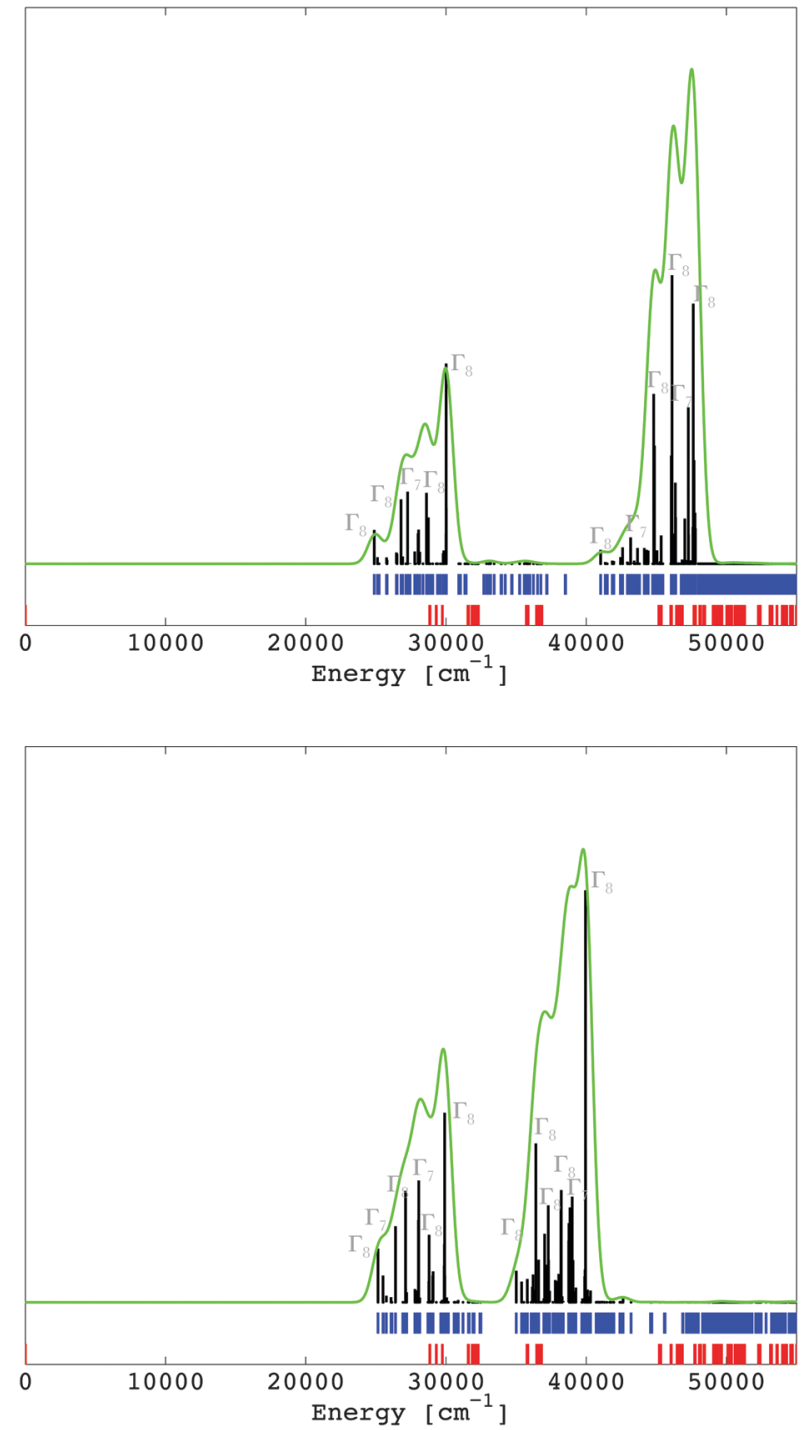

Fig. 7 Calculated multiplet energy levels from the $4 f^{7}$ (in red) and $4 f^{6} 5 d^{1}$ (in blue) configurations of $\mathrm{Eu}^{2+}$ in $\mathrm{CaF}_{2}$ (up) and $\mathrm{SrCl}_{2}$ (down), together with the intensities of the excitation $4 f^{7} \rightarrow 4 f^{6} 5 d^{1}$ transitions, i.e. zero phonon lines (in black). The green curve represents a superimposition of a Gaussian band with a width of $500 \mathrm{~cm}^{-1}$ on the zero phonon lines.

absolute value, if we consider an arbitrary scale of spectral rendering. The zero-field splitting, which transforms the ${ }^{8} \mathrm{~S}_{7 / 2}$ state of $4 \mathrm{f}^{7}$ to $\Gamma_{6}+\Gamma_{7}+\Gamma_{8}$ in the actual octahedral symmetry, is in the magnitude of tenths of $\mathrm{cm}^{-1}$. The $4 \mathrm{f}^{6} 5 \mathrm{~d}^{1}$ transitions are characterized by two dominant bands (Fig. 7), in line with the excitation spectrum seen in ref. 41 and 42 for $\mathrm{CaF}_{2}: \mathrm{Eu}^{2+}$ and $\mathrm{SrCl}_{2}: \mathrm{Eu}^{2+}$. The correspondence between the theoretical results and the excitation spectrum is seen in the ESI $\neq$, where the excitation spectra of $\mathrm{CaF}_{2}: \mathrm{Eu}^{2+}$ (Fig. S1, ESI $\ddagger$ ) and $\mathrm{SrCl}_{2}: \mathrm{Eu}^{2+}$ (Fig. S2, ESI $\$$ ) are reproduced from ref. 41 and 42 .

\section{Conclusions}

Optical and magnetic effects in lanthanide-based compounds are phenomena intimately understood with the help of ligand 
field theory. In this work, we have outlined important points for a realistic description of the electronic structure and the optical properties of $\mathrm{Eu}^{2+}$-doped $\mathrm{CaF}_{2}$ and $\mathrm{SrCl}_{2}$ compounds. The treatment of the local distortions due to the presence of the $\mathrm{Eu}^{2+}$ impurity in the fluorite structures of $\mathrm{CaF}_{2}$ and $\mathrm{SrCl}_{2}$ was addressed by periodical band structure calculation. The LFDFT algorithm was used for the calculation of the multiplet energy levels of the $4 \mathrm{f}^{7}$ and $4 \mathrm{f}^{6} 5 \mathrm{~d}^{1}$ electron configurations of $\mathrm{Eu}^{2+}$. The optical $4 \mathrm{f}^{7}-4 \mathrm{f}^{6} 5 \mathrm{~d}^{1}$ transitions were determined, with a good qualitative agreement achieved between the non-empirical investigations and the experimental findings. In particular, the convoluted calculated spectrum can be immediately compared with experimental data, thus showing the usefulness of the approach to experimental scientists. The computational methods and post-computational analyses contained in the LFDFT algorithm are producing reliable ligand field and related parameters, consolidating the academic insight into the structure-property relationships of rare-earth materials and paving the way to the desiderata of property engineering.

There are several advantageous characteristics that this fully non-empirical LFDFT method possesses which should be noted and remembered, besides the predictive capability, very important today for the vast number and kind of rare-earth-based technological materials. The method can be applied to any lanthanide ions, for general $4 \mathrm{f}^{n}-4 \mathrm{f}^{n-1} 5 \mathrm{~d}^{1}$ transitions with different coordination symmetries. The LFDFT approach has other advantages against widespread semi-empirical and full $a b$ initio methods, not least the fact that it can be applied to larger-size systems in a relatively short computational time.

\section{Acknowledgements}

This work is supported by the Swiss National Science Foundation (SNF) and the Swiss State Secretariat for Innovation and Research. Support from the UEFISCDI Romania research grant PCE 14/2013 from the Romanian Ministry of Research is also acknowledged.

\section{Notes and references}

1 H. Bethe, Ann. Phys., 1929, 295, 133.

2 J. H. Van Vleck, J. Chem. Phys., 1935, 3, 807.

3 J. S. Griffith, The Theory of Transition Metal Ions, Cambridge University Press, Cambridge, 1961.

4 M. Gerloch, J. H. Harding and R. G. Woodley, Struct. Bonding, 1981, 46, 1.

5 B. N. Figgis and M. A. Hitchman, Ligand Field Theory and Its Applications, Wiley-VCH, New York, 2000.

6 C. Schäffer and C. Jørgensen, Mol. Phys., 1965, 9, 401.

7 W. Urland, Chem. Phys., 1976, 14, 393.

8 C. Daul, Int. J. Quantum Chem., 1994, 52, 867.

9 C. Daul, E. J. Baerends and P. Vernooijs, Inorg. Chem., 1994, 33, 3538.

10 M. Atanansov, C. A. Daul and C. Rauzy, Struct. Bonding, 2004, 106, 97.
11 L. Noodleman and J. G. Norman, J. Chem. Phys., 1979, 70, 4903.

12 L. Noodleman, J. Chem. Phys., 1981, 74, 5737.

13 M. Mitani, V. Mori, Y. Takano, D. Yamaki, Y. Yoshioka and K. Yamaguchi, Chem. Phys., 2000, 113, 4035.

14 A. Bencini and F. Totti, Int. J. Quantum Chem., 2005, 101, 819. 15 F. Cimpoesu, B. Frescus, C. I. Oprea, H. Ramanantoanina, W. Urland and C. Daul, Mol. Phys., 2015, DOI: 10.1080/ 00268976.2015.1007107.

16 M. Atanasov, C. Rauzy, P. Baettig and C. Daul, Int. J. Quantum Chem., 2005, 102, 119.

17 M. Atanasov, C. Daul, H. U. Gudel, T. A. Wesolowski and M. Zbiri, Inorg. Chem., 2005, 44, 2954.

18 D. Reinen, M. Atanasov and W. Massa, Z. Anorg. Allg. Chem., 2006, 632, 1375.

19 M. Atanasov, P. Comba and C. Daul, Inorg. Chem., 2008, 47, 2449. 20 M. Gruden-Pavlovic, M. Peric, M. Zlatar and P. Garcia-Fernandez, Chem. Sci., 2014, 5, 1453.

21 Z. D. Matovic, M. S. Jeremic, R. M. Jelic, M. Zlatar and I. Z. Jakovljevic, Polyhedron, 2013, 55, 131.

22 G. Morrison, H. Ramanantoanina, W. Urland, M. D. Smith and H.-C. zur Loye, Inorg. Chem., 2015, 54, 5504-5511.

23 C. A. Daul, J. Phys.: Conf. Ser., 2013, 428, 012023.

24 A. Borel, C. A. Daul and L. Helm, Chem. Phys. Lett., 2004, 383, 584.

25 F. Senn, L. Helm, A. Borel and C. A. Daul, C. R. Chim., 2012, 15, 250.

26 M. Atanasov and C. A. Daul, Chem. Phys. Lett., 2003, 379, 209.

27 M. Atanasov, P. Comba and C. Daul, Inorg. Chem., 2006, 110, 13332.

28 I. Ciofini and C. Daul, Coord. Chem. Rev., 2003, 238-239, 187.

29 M. Atanasov and C. A. Daul, C. R. Chim., 2005, 8, 1421.

30 M. Atanasov, E. J. Baerends, P. Bruyndonckx, C. Daul, C. Rauzy and M. Zbiri, Chem. Phys. Lett., 2004, 399, 433.

31 F. Senn and C. A. Daul, THEOCHEM, 2010, 954, 105.

32 F. Senn, M. Zlatar, M. Gruden-Pavlovica and C. Daul, Monatsh. Chem., 2011, 142, 593.

33 L. Petit, A. Borel, C. Daul, P. Maldivi and C. Adamo, Inorg. Chem., 2006, 45, 7382 .

34 M. Atanasov and C. Daul, Chimia, 2005, 59, 504.

35 H. Ramanantoanina, W. Urland, F. Cimpoesu and C. Daul, Phys. Chem. Chem. Phys., 2013, 15, 13902.

36 H. Ramanantoanina, W. Urland, A. Garcia-Fuente, F. Cimpoesu and C. Daul, Chem. Phys. Lett., 2013, 588, 260.

37 S. Nakamura and G. Fasol, The Blue Laser Diode, Springer, Berlin, 1997.

38 H. Ramanantoanina, W. Urland, A. Garcia-Fuente, F. Cimpoesu and C. Daul, Phys. Chem. Chem. Phys., 2014, 16, 14625.

39 H. Ramanantoanina, W. Urland, B. Herden, F. Cimpoesu and C. Daul, Phys. Chem. Chem. Phys., 2015, 17, 9116.

40 B. Herden, A. Garcia-Fuente, H. Ramanantoanina, T. Jüstel, C. Daul and W. Urland, Chem. Phys. Lett., 2015, 620, 29.

41 G. W. Burdick, A. Burdick, V. Deev, C.-K. Duan and M. F. Reid, J. Lumin., 2006, 118, 205.

42 Z. Pan, L. Ning, B.-M. Cheng and P. A. Tanner, Chem. Phys. Lett., 2006, 428, 78. 
43 R. D. Cowan, The theory of atomic structure and spectra, University of California Press, Berkeley, 1981.

44 H. Lueken, Magnetochemie, Teubner Studienbücher Chemie, Stuttgart, 1999.

45 M. Gerloch, Magnetism, ligand-field analysis, Cambridge University Press, Cambridge, 1983.

46 S. Hüfner, Optical spectra of transparent rare earth compounds, Academic Press, New York, 1978.

47 H. Ramanantoanina, W. Urland, F. Cimpoesu and C. Daul, Phys. Chem. Chem. Phys., 2014, 16, 12282.

48 G. te Velde, F. M. Bickelhaupt, S. J. A. van Gisbergen, C. F. Guerra, E. J. Baerends, J. G. Snijders and T. Ziegler, J. Comput. Chem., 2001, 22, 931.

49 C. F. Guerra, J. G. Snijders, G. te Velde and E. J. Baerends, Theor. Chem. Acc., 1998, 99, 391.

50 E. J. Baerends, T. Ziegler, J. Autschbach, D. Bashford, A. Berces, F. M. Bickelhaupt, C. Bo, P. M. Boerrigter, L. Cavallo, D. P. Chong, L. Deng, R. M. Dickson, D. E. Ellis, M. van Faassen, L. Fan, T. H. Fischer, C. F. Guerra, A. Ghysels, A. Giammona, S. J. A. van Gisbergen, A. W. Götz, J. A. Groeneveld, O. V. Gritsenko, M. Grüning, S. Gusarov, F. E. Harris, P. van den Hoek, C. R. Jacob, H. Jacobsen, L. Jensen, J. W. Kaminski, G. van Kessel, F. Koostra, A. Kovalenko, M. V. Krykunov, E. van Lenthe, D. A. McCormack, A. Michalak, M. Mitoraj, J. Neugebauer, V. P. Nicu, L. Noodleman, V. P. Osinga, S. Patchkovskii, P. H. T. Philipsen, D. Post, C. C. Pye, W. Ravenek, J. I. Rodriguez, P. Ros, P. R. T. Shipper, G. Schreckenbach, J. S. Seldenthuis, M. Seth, J. G. Snijders, M. Sola, M. Swart, D. Swerhone, G. te Velde, P. Vernooijs, L. Versluis, L. Vissher, O. Visser, F. Wang, T. Wesolowski, E. M. van Wezenbeek, G. Wiesenekker, S. K. Wolff, T. K. Woo, A. L. Yarkolev, ADF2013.01, available at http://www.scm.com.

51 P. J. Stephens, F. J. Devlin, C. F. Chabalowski and M. J. Frisch, J. Phys. Chem., 1994, 98, 11623.

52 (a) G. Kresse and J. Hafner, Phys. Rev. B: Condens. Matter Mater. Phys., 1993, 47, 558; (b) G. Kresse and J. Furthmüller, Phys. Rev. B: Condens. Matter Mater. Phys., 1996, 54, 11169.

53 S. H. Vosko, L. Wilk and M. Nussair, Can. J. Phys., 1980, 58, 1200.

54 J. P. Perdew, K. Burke and M. Ernzerhof, Phys. Rev. Lett., 1996, 77, 3865.

55 P. E. Blöchl, Phys. Rev. B: Condens. Matter Mater. Phys., 1994, 50, 17953.

56 G. Kresse and D. Joubert, Phys. Rev. B: Condens. Matter Mater. Phys., 1999, 59, 1758.

57 T. Ziegler, A. Rauk and E. J. Baerends, Theor. Chim. Acta, 1977, 43, 261.

58 See also the two different approaches devoted to the calculation of the Slater-Condon parameters in the two-openshell $4 \mathrm{f}^{2}$ and $4 \mathrm{f}^{1} 5 \mathrm{~d}^{1}$ configurations of $\mathrm{Pr}^{3+}$ in ref. 35 and 39.

59 P. Pyykkö and J. P. Desclaux, Acc. Chem. Res., 1979, 12, 276. 60 K. S. Pitzer, Acc. Chem. Res., 1979, 12, 271.
61 C. Clavaguera, J.-P. Dognon and P. Pyykkö, Chem. Phys. Lett., 2006, 429, 8.

62 P. Pyykkö, Chem. Rev., 1988, 88, 563.

63 P. Pyykkö, Chem. Rev., 2012, 112, 371.

64 D. Cremer, W. Zou and M. Filatov, WIREs Comput. Mol. Sci., $2014,4,436$.

65 E. van Lenthe, A. E. Ehlers and E. J. Baerends, J. Chem. Phys., 1999, 110, 8943.

66 E. van Lenthe, E. J. Baerends and J. G. Snijders, J. Chem. Phys., 1993, 99, 4597.

67 E. van Lenthe, E. J. Baerends and J. G. Snijders, J. Chem. Phys., 1993, 101, 9783.

68 E. van Lenthe, J. G. Snijders and E. J. Baerends, J. Chem. Phys., 1996, 105, 6505.

69 E. van Lenthe, R. van Leeuwen, E. J. Baerends and J. G. Snijders, Int. J. Quantum Chem., 1996, 57, 281.

70 J. G. Snijders and E. J. Baerends, Mol. Phys., 1978, 36, 1789. 71 J. G. Snijders, E. J. Baerends and P. Ros, Mol. Phys., 1979, 38, 1909.

72 T. Ziegler, J. G. Snijders and E. J. Raerends, J. Chem. Phys., 1981, 74, 1271.

73 T. Ziegler, V. Tschinke, E. J. Baerends, J. G. Snijders and W. Ravenek, J. Phys. Chem., 1989, 93, 3050.

74 P. M. Boerrigter, M. A. Buijse and J. G. Snijders, Chem. Phys., 1988, 122, 357.

75 R. D. Cowan and D. C. Griffin, J. Opt. Soc. Am., 1976, 66, 1010. 76 A. Kramida, Y. Ralchenko, J. Reader and NIST ASD Team, NIST Atomic Spectra Database (ver. 5.2), [Online], National Institute of Standards and Technology, Gaithersburg, MD, 2014, available: http://physics.nist.gov/asd, April 21, 2015.

77 C. W. Nielson and G. F. Koster, Spectroscopic Coefficients for the $p^{n}, d^{n}$ and $f^{n}$ Configurations, The MIT Press Cambridge, 1963.

78 R. E. Walstedt and L. R. Walker, Phys. Rev. B: Solid State, 1974, 9, 4857.

79 P. A. O'Day, J. J. Rehr, S. I. Zabinsky and G. E. Brown, J. Am. Chem. Soc., 1994, 116, 2938.

80 P. Ghina, A. Carollo, G. Flor, L. Malavasi and G. S. Peruga, J. Phys. Chem. B, 2005, 109, 4365.

81 B. V. Padlyak, M. Grinberg, T. Lukasiewicz, J. Kisielewski and M. swirkowicz, J. Alloys Compd., 2003, 361, 6.

82 D. N. Batchelder and R. O. Simmons, J. Chem. Phys., 1964, 41, 2324.

83 H. Ott, Z. Kristallogr., 1926, 63, 222.

84 C. K. Jørgensen, Absorption Spectra and Chemical Bonding in Complexes, Pergamon Press, Oxford, 1962.

85 P. A. Tanner and Y. Y. Yeung, J. Phys. Chem. A, 2013, 117, 10726.

86 D. L. Clark, J. C. Gordon, P. J. Hay and R. Poli, Organometallics, 2005, 24, 5747.

87 F. Cimpoesu, N. Dragoe, H. Ramanantoanina, W. Urland and C. Daul, Phys. Chem. Chem. Phys., 2014, 16, 11337.

88 M. L. Neidig, D. L. Clark and R. L. Martin, Coord. Chem. Rev., 2013, 257, 394. 